\section{Multiple Septic Bursitis and Spontaneous Achilles Tendon Tear in Systemic Lupus Erythematosus}

\section{To the Editor:}

We describe a 31-year-old woman who was admitted because of fever and polyarthritis of 1 week's duration. Six months before, she was diagnosed with systemic lupus erythematosus (SLE) on the basis of nonerosive polyarthritis, painless oral ulcers, positive antinuclear antibody (IFI-Hep2) titer of 1:10,240 with homogeneous pattern, and anti-dsDNA (Farr assay) value of $146 \mathrm{IU} / 1$ (normal 0-7 IU/1). At admission she denied any dyspnea, chest pain, rash, dysuria, diarrhea, or intravenous drug abuse. She was receiving methylprednisolone $32 \mathrm{mg} / \mathrm{qd}$, hydroxychloroquine $400 \mathrm{mg} / \mathrm{qd}$, and methotrexate $15 \mathrm{mg} /$ week plus folic acid, but she also reported that in the last month her doctor prescribed a course of 3 consecutive daily pulses of methylprednisolone $500 \mathrm{mg}$, because of exacerbation of articular manifestations. On examination she presented with hyperpyrexia (up to $40^{\circ} \mathrm{C}$ ), shaking chills, and severe inflammation of the left ankle retrocalcaneal region and the bilateral metatarsophalangeal joints (Figure 1). Musculoskeletal manifestations appeared to be out of proportion to SLE disease activity. Heart and chest examinations were unremarkable. She reported itching and painful defecation without blood-streaked stools and mucopurulent discharge.

Perineal inspection demonstrated perianal fissures and erythema, suggesting a bacterial skin infection, in the absence of any anorectal abscess or fistula. Routine laboratory tests showed erythrocyte sedimentation rate $52 \mathrm{~mm} / \mathrm{h}$, C-reactive protein $14.4 \mathrm{mg} / \mathrm{dl}$ (normal 0-0.5 mg/dl), white blood cell count $16,000 / \mathrm{mm}^{3}$ (95\% neutrophils), anti-dsDNA $16 \mathrm{IU} / 1$ (normal 0-7), and normal levels of $\mathrm{C} 3$ and $\mathrm{C} 4$ complement fractions. Because of the high suspicion of sepsis, piperacillin/tazobactam intravenously (IV) $4 \mathrm{~g}$ $+0.5 \mathrm{~g} /$ tid plus teicoplanin IV $400 \mathrm{mg}$ /day were started on the first day after starting microbiological tests.

Tests for the human immunodeficiency virus, Epstein-Barr virus, and cytomegalovirus infection were all negative as well as urine, stool, pharyngeal, and vaginal cultures. Gram-positive cocci growth was revealed by culture of the perianal lesion and Staphylococcus aureus non-methicillin-resistant was isolated from both blood and left subcutaneous calcaneal bursa drainage cultures. The antibiogram showed no antibiotic resistance of the isolated bacterial strain and confirmed the sensitivity of bacteria to the administered antibiotics. Electrocardiography, chest radiographs, and abdominal ultrasound showed normal findings. In addition, a Doppler transthoracic echocardiogram excluded the presence of infective endocarditis and revealed a preserved ejection fraction (60\%) and normal endocardium and pericardium. Ultrasound examination of the feet and magnetic resonance imaging of the left ankle showed normal joint findings and supported the diagnosis of multiple septic bursitis with left Achilles tendon tear (Figures 2 and 3).

One week later, the patient was still feverish. Repeated Doppler transthoracic echocardiogram showed normal findings, blood culture results were negative, but the left subcutaneous calcaneal bursa drainage culture was positive for $S$. aureus non-methicillin-resistant again. Hence, 2 weeks after admission, according to the antibiogram, treatment was substituted with cefepime IV $2 \mathrm{~g} / \mathrm{bid}$ and gentamicin IV $80 \mathrm{mg} / \mathrm{tid}$, which allowed complete resolution of sepsis in 2 weeks' time. After 2 months, our patient is free from any recurrence of infection, SLE is well controlled by her previous therapy, and steroid tapering is scheduled.

Infections are among the most important causes of morbidity and mortality in patients with SLE, being responsible for approximately $25 \%$ of all deaths, and their prevalence appears to be highest within the first 5 years of disease onset ${ }^{1}$. The risk of major infections is influenced by treatment, mainly corticosteroids, even at moderate doses, with each $10 \mathrm{mg}$ per day of prednisone increasing the risk $11-$ fold $^{2}$. Bacteremia accounts for $24 \%$ of the major infections in patients with SLE while polyarticular septic complications are extremely rare ${ }^{2,3}$. Joint or bursa infection, in which the most frequent causative organisms identified are $S$. aureus, can derive from hematogenous spread or from direct dissemination after trauma or invasive procedures, especially in immunosuppressed patients and in a damaged joint or bursa ${ }^{4}$. Spontaneous tendon rupture in patients with SLE is a rare but potentially disabling complication. Alves, $e t a l^{5}$ highlighted that corti-

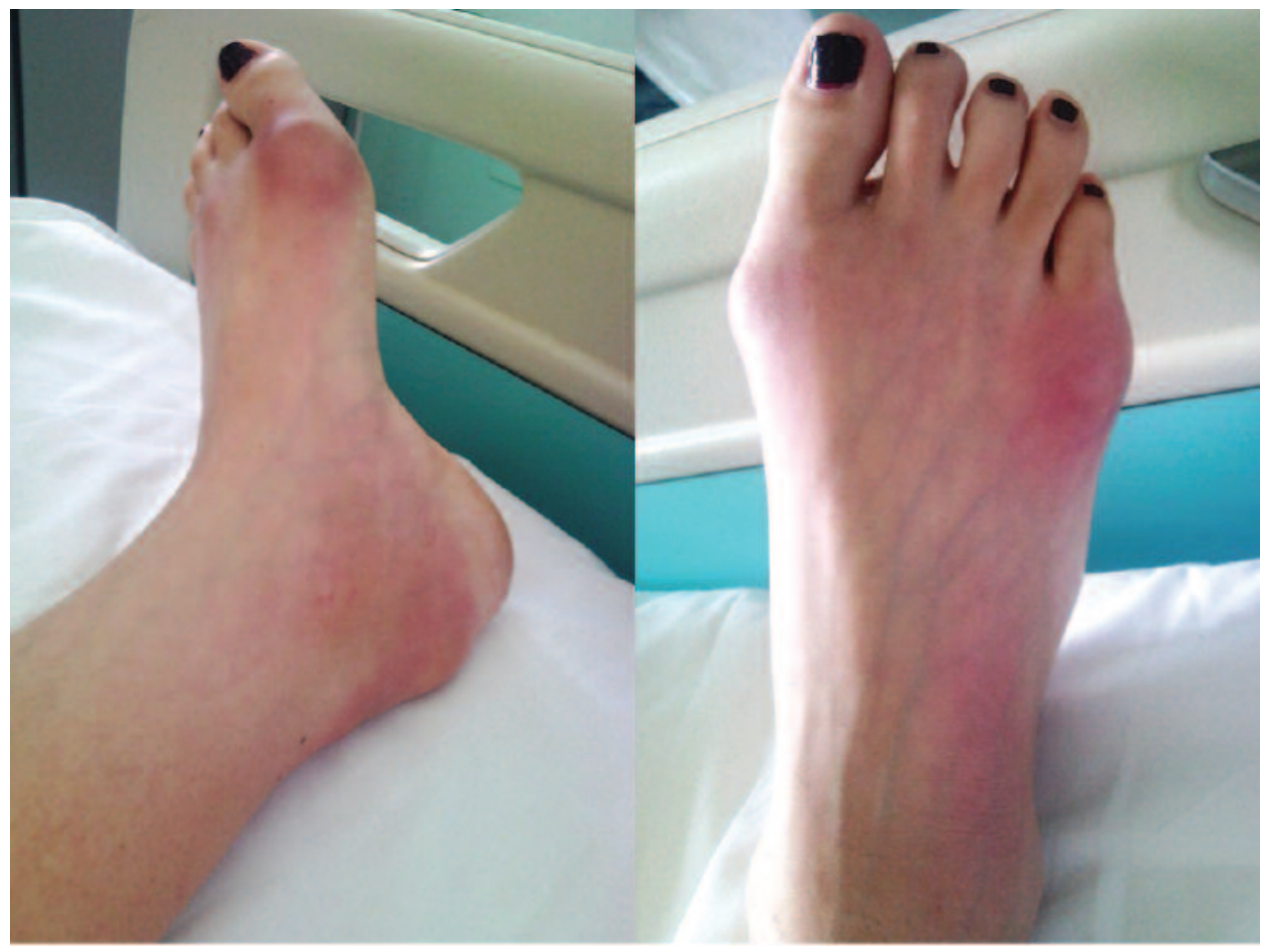

Figure 1. Erythema and swelling of the left retrocalcaneal region, the left first and third, and the right first and fifth metatarsophalangeal joints. 


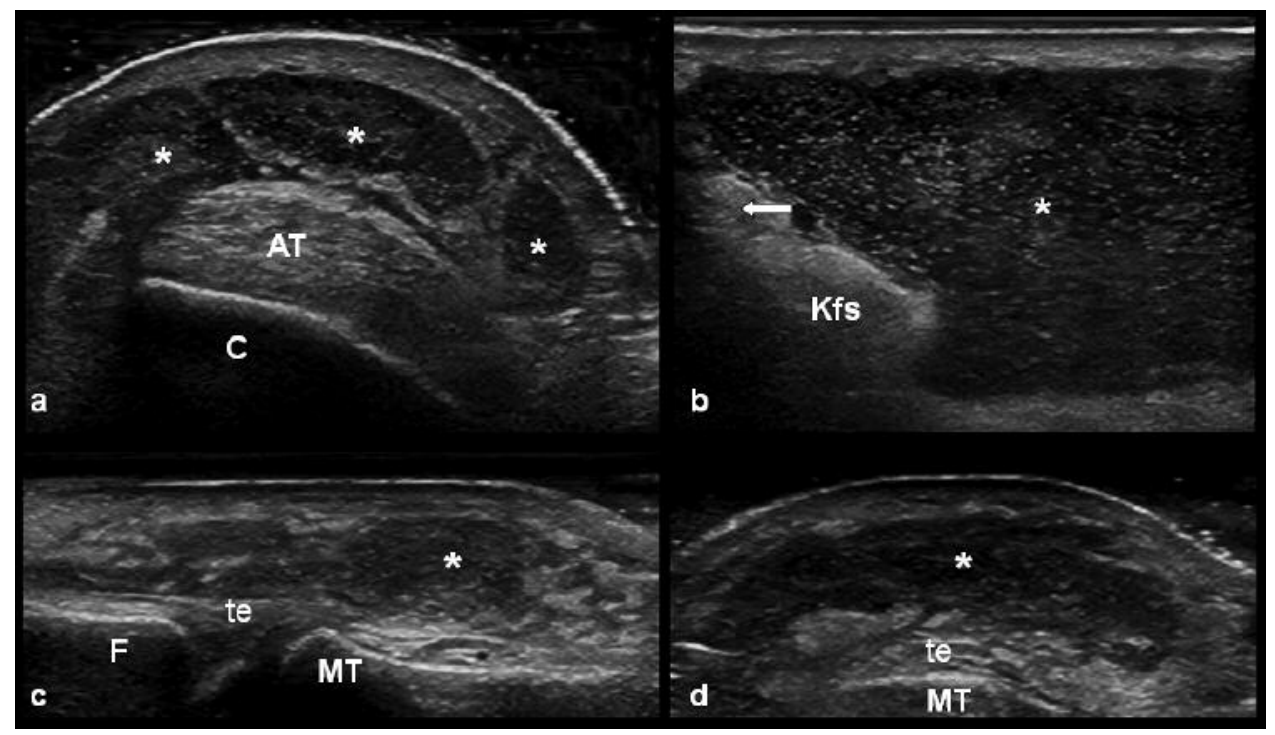

Figure 2. Transverse (A) and longitudinal (B) ultrasonography images of the retrocalcaneal region show a large hypoechoic area (*) filled with echogenic particulate material in the region of the subcutaneous bursa. Arrow indicates distal end of the ruptured Achilles tendon. Transverse (C) and longitudinal (D) images of the fifth right metatarsophalangeal joint lateral surface show an area $(*)$, corresponding to the bursa that intervenes between the tendon of abductor digiti minimi and the head of the fifth metatarsal, ranging from hypoechoic to hyperechoic, due to the coexistence of fluid and debris found in septic bursitis. C: calcaneus; AT: Achilles tendon; MT: metatarsal head; F: phalanx; te: tendon; Kfs: Kager's fat pad.

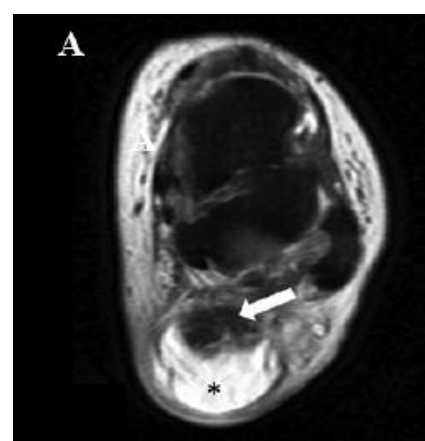

B
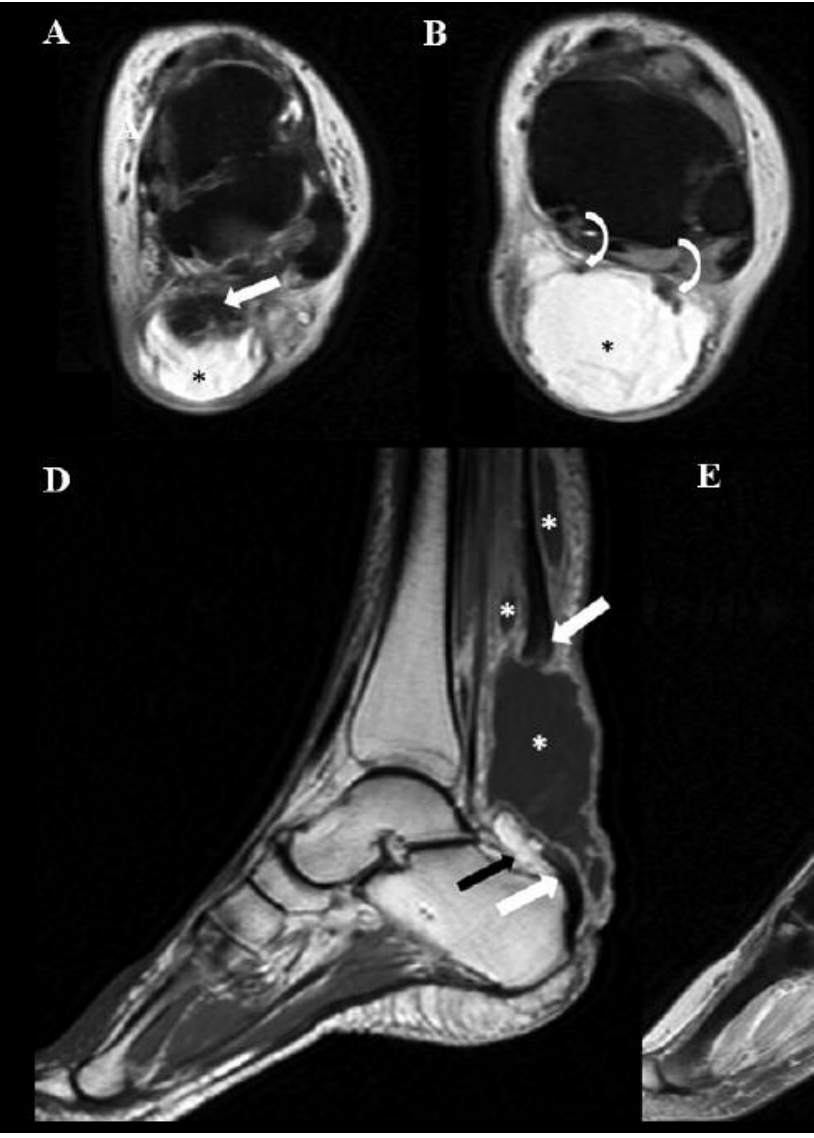

C

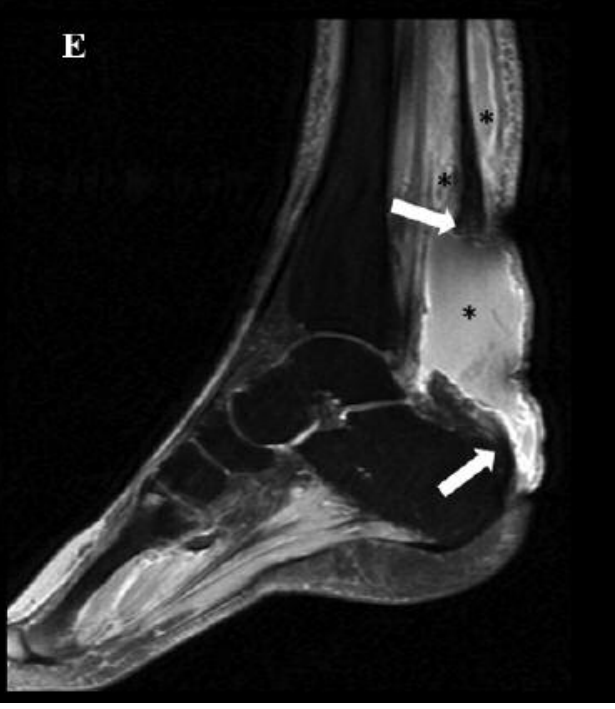

Figure 3. MRI of left ankle. Axial proton density spectral presaturation inversion recovery (SPIR) images (A, B, and C) show a bright signal intensity lesion $(*)$, extending from the retrocalcaneal region (A) to over the distal tibial metaphysis (C). It surrounds the Achilles tendon (white arrows), which appears enlarged, not homogeneous and subtotally torn (B, curved white arrows). Sagittal T1-weighted image (D) and DP SPIR image (E) after IV administration of gadolinium contrast material show a localized fluid collection with intermediate to low signal intensity $(*)$, which enhances peripherally and lacks enhancement centrally, found in soft tissue abscess likely originating from the subcutaneous bursa. There is clear partition between the fluid collection and Kager's fat pad (black arrow), which appears spared by the septic process (D). White arrows show distal and proximal ends of Achilles tendon (E). 
costeroid therapy and Jaccoud's arthropathy are risk factors for tendon tear, and the most frequently observed rupture sites are the patellar and Achilles tendons.

In our patient it is likely that septicemia originated from the perianal skin infection, which is considered an important risk factor for septic arthritis in patients with joint disease ${ }^{6}$, and the infection affected bursae, causing the development of retrocalcaneal abscess, which facilitated the Achilles tendon rupture.

Previous reports focused on the risk of single septic bursitis in SLE as a manifestation of the general susceptibility to infections observed in patients with $\mathrm{SLE}^{7,8}$. In our case, the high doses of corticosteroids might have favored both infection development and spreading. To our knowledge this is the first report of multiple septic bursitis in a patient with SLE. Because multiple septic bursitis may mimic or coexist with SLE symptoms, clinicians should be aware of its occurrence.

MATTEO PIGA, MD, Research Fellow; ALESSANDRA GABBA, MD, Research Fellow; ALESSANDRA VACCA, MD; PIETRO GARAU, MD; VALENTINA IBBA, MD; ALESSANDRO MATHIEU, MD, Professor, Chief, Chair of Rheumatology and Rheumatology Unit; University and AOU of Cagliari, SS 554-09-042 Monserrato, Cagliari, Italy. Address correspondence to Dr. Piga; E-mail: matteopiga@alice.it

\section{REFERENCES}

1. Cervera R, Khamashta MA, Font J, Sebastiani GD, Gil A, Lavilla $\mathrm{P}$, et al. Morbidity and mortality in systemic lupus erythematosus during a 10-year period: a comparison of early and late manifestations in a cohort of 1,000 patients. Medicine 2003;82:299-308.

2. Ruiz-Irastorza G, Olivares N, Ruiz-Arruza I, Martinez-Berriotxoa A, Egurbide MV, Aguirre C. Predictors of major infections in systemic lupus erythematosus. Arthritis Res Ther 2009;11:R109.

3. Dubost JJ, Fis I, Denis P, Lopitaux R, Soubrier M, Ristori JM, et al. Polyarticular septic arthritis. Medicine 1993;72:296-310.

4. Mathews CJ, Weston VC, Jones A, Field M, Coakley G. Bacterial septic arthritis in adults. Lancet 2010;375:846-55.

5. Alves EM, Macieira JC, Borba E, Chiuchetta FA, Santiago MB. Spontaneous tendon rupture in systemic lupus erythematosus: association with Jaccoud's arthropathy. Lupus 2010;19:247-54 .

6. Kaandorp CJ, Van Schaardenburg D, Krijnen P, Habbema JD, van de Laar MA. Risk factors for septic arthritis in patients with joint disease. A prospective study. Arthritis Rheum 1995;38:1819-25.

7. Greene R, Kaufman R, Quismorio FP Jr. Septic bursitis in systemic lupus erythematosus. Clin Rheumatol 1984;3:55-9.

8. Lambie P, Kaufman R, Beardmore T. Septic ischial bursitis in systemic lupus erythematosus presenting as a perirectal mass. J Rheumatol 1989;16:1497-9.

J Rheumatol 2010;37:11; doi:10.3899/jrheum.100409 